\title{
Liquen plano oral (I). Aspectos clínicos, etiopatogénicos y epidemiológicos
}

\author{
Bascones-llundain $C^{*}$, González Moles MA**, Carrillo de Albornoz A***, \\ Bascones-Martínez A****
}

\section{RESUMEN}

Se presenta un estudio clínico, etiopatogénico y epidemiológico de una muestra amplia de pacientes con liquen plano oral evaluados en la Facultad de Odontología de la Universidad Complutense de Madrid.

Palabras clave: Liquen plano oral, mucosa oral.

\section{SUMMARY}

An etiopathogenic and epidemiological clinical study conducted in a broad group of patients with oral lichen planus was evaluated at the Faculty of Dentistry at the Complutense University of Madrid.

Key words: Oral lichen planus, oral mucous membrane.

Aceptado para publicación:

* Doctora en Odontología y Master por la Universidad Complutense de Madrid.

** Profesor Titular. Facultad de Odontología. Universidad de Granada.

*** Alumna de doctorado. Facultad de Odontología. Universidad Complutense de Madrid.

**** Catedrático. Facultad de Odontología. Universidad Complutense de Madrid.

Bascones-Ilundain C, González Moles MA, Carrillo de Albornoz A, Bascones-Martínez A. Liquen plano oral (I). Aspectos clínicos, etiopatogénicos y epidemiológicos. Av. Odontoestomatol 2006; 22-1: 11-19.

\section{INTRODUCCIÓN}

El liquen plano oral (LP) es una enfermedad mucocutánea crónica, de carácter inflamatorio, etiología desconocida y naturaleza autoinmune, en la que se produce una agresión $\mathrm{T}$ linfocitaria dirigida frente a las células basales del epitelio de la mucosa oral. (1-3)

Las células atacadas desarrollan un complejo mecanismo molecular enfocado a detener el ciclo celular de la reparación del DNA (4) o inducir la apoptosis con el fin de eliminar células muy dañadas en su DNA (5). Algunos autores $(6,7)$ afirman que las células epiteliales del LPO frecuentemente responden a este ataque con un aumento en las tasas de proliferación.

Sería posible afirmar que los mecanismos moleculares que controlan el crecimiento, proliferación, maduración y apoptosis en las células epiteliales atacadas pueden jugar un papel importante en el proceso de transformación maligna. 
Clínicamente se manifiesta con formas reticulares o atrófico-erosivas y potencialmente puede afectar a la piel, anejos cutáneos y mucosas, siendo frecuente que las lesiones bucales precedan a las cutáneas o que sea única la localización intraoral (8). El liquen plano es considerado una condición precancerosa, aunque presenta cierta controversia al respecto (9). Las manifestaciones orales parecen ser las mas frecuentes habiéndose propuesto a lo largo de los años multitud de clasificaciones clínicas.

La variante reticular es la más frecuente apareciendo como una lesión blanca, lineal en forma estrellada, de retícula, rama o anular ligeramente sobreelevada, palpable (estrías de Wickham) y que no se desprende. Se presenta en zonas amplias de la mucosa yugal preferentemente de manera bilateral, simétrica y asintomática $(10,11)$.

La variante atrófica-erosiva se caracteriza por un adelgazamiento del espesor del epitelio manifestándose por un enrojecimiento generalizado y difuso exponiéndose los vasos sanguíneos subyacentes del corion. La localización más frecuente es la lengua siendo también la encía un lugar habitual de asentamiento presentándose como una gingivitis descamativa crónica. Esta forma se caracteriza por la presencia de ulceraciones del epitelio sobre una mucosa atrófica, aunque también podría presentarse como forma ampollosa. (Figuras 1 y 2).

Desde el punto de vista clínico se trata de una afectación mucocutánea que puede manifestarse en cuero cabelludo, piel, uñas y mucosas. La variante cutánea se caracteriza por lesiones en forma de pápulas de 2-4 mm. de diámetro, en forma poligonal que aparecen de manera aislada o agrupándose a manera de placas diferenciadas de la piel que las rodea. Al principio tienen color rosado que después pasa a violáceo y finalmente a color pardo. Las localizaciones más frecuentes son las áreas flexoras y especialmente la cara anterior de las muñecas (8).

No se ve diferencia en el mecanismo patogénico entre el LPO reticular y el atrófico-erosivo (12) aunque histológicamente sí que se observa a nivel del corion diferencia en la densidad del infiltrado inflamatorio siendo mayor en los atrófico erosivos que en los reticulares (13).
El diagnóstico del LPO debe basarse en la observación clínica y confirmarse con la descripción de las características histopatológicas. Hay una serie de criterios clínicos e histopatológicos importantes para sentar el diagnóstico de una manera concluyente (14).

El patrón clínico característico es la presencia de lesiones bilaterales más o menos simétricas y de líneas blanquecinas en el patrón reticular o lesiones atróficas en la variante atrófico-erosivo.

Los criterios histopatológicos son la presencia de una banda bien definida de infiltrado inflamatorio compuesta principalmente por linfocitos, la degeneración hidrópica de la capa basal y la ausencia de displasia.

Si estas características no son obvias se debe hablar de lesión compatible con LPO.

En el diagnóstico final de LPO y de reacción liquenoide (RL) se deben incluir tanto el diagnóstico clínico como el histológico.

El término RL se utilizará bajo las siguientes condiciones: clínica compatible con LPO e histológicamente es un LPO, clínicamente compatible con LPO e histológicamente también compatible con el mismo (14).

En la anamnesis se debe preguntar por los antecedentes familiares y personales y en especial si toma algún fármaco, su estado psicológico y una analítica si fuera necesario. En relación con la historia natural de la lesión se debe incidir desde cuando la tiene, si suele desaparecer o cambiar de lugar, la respuesta ante tratamientos anteriores, dolor en caso de ser una variante roja, quemazón y sangrado espontáneo o al cepillarse. Por último se debe investigar sobre la presencia de otras localizaciones extraorales tanto mucosas como cutáneas (11).

En la exploración hay que ver su localización, siendo la más frecuente dentro de la cavidad bucal la mucosa yugal, lengua y encía, aunque también se suele encontrar en la mucosa labial, suelo de boca, comisura labial y paladar. En la mucosa yugal se presenta de forma bilateral y simétrica. Al explorar las manifestaciones cutáneas se deben observar las áreas flexoras y dentro de ellas la cara anterior de las muñe- 

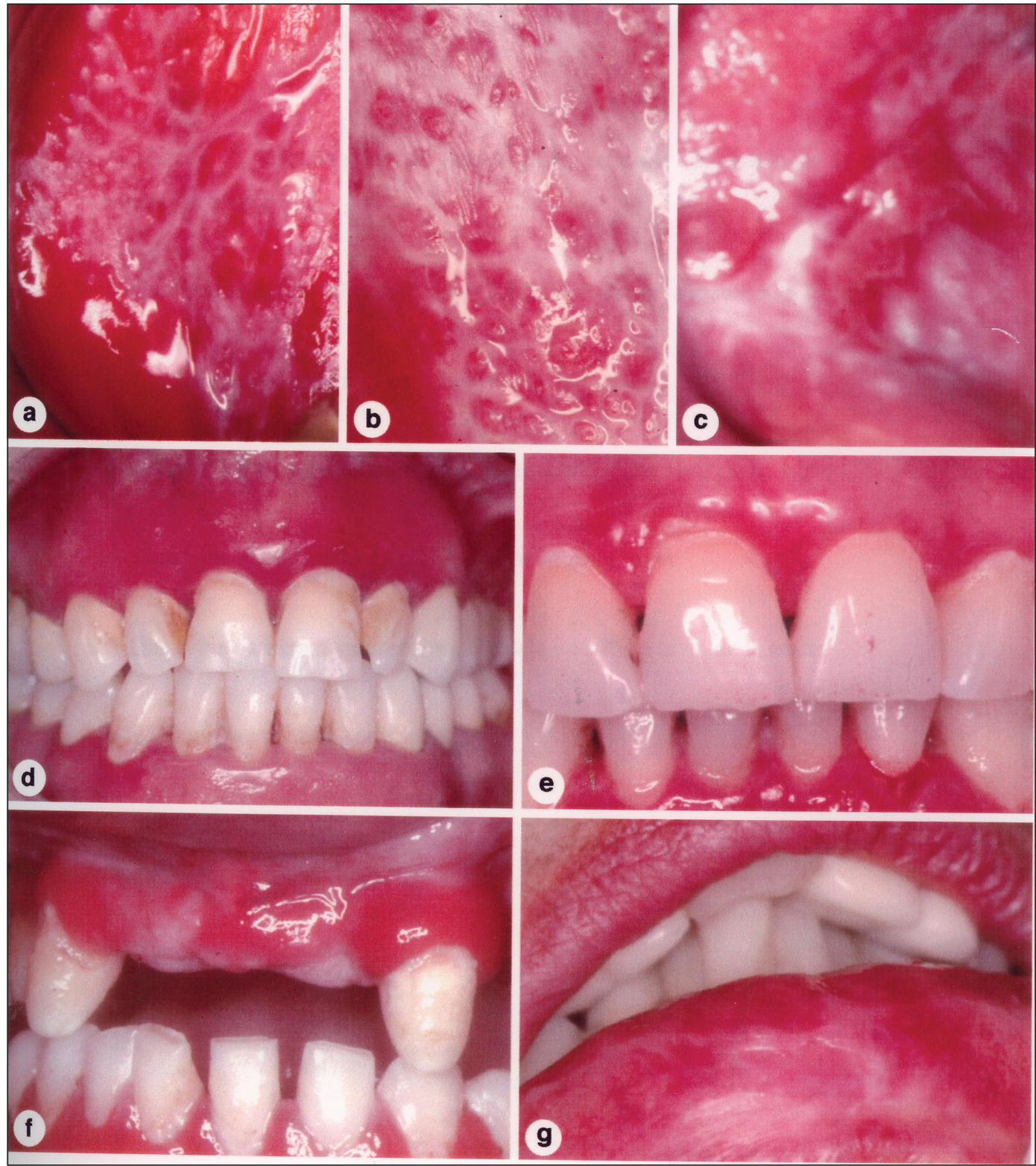

Fig. 1. a. Estrías de

Wickham de un liquen plano reticular localizado en la mucosa yugal retrocomisural;

b. Estrías de Wickham de un LPO reticular en la mucosa de la mejilla;

c. Liquen erosivo ulcerado en la mucosa de la mejilla; d. Gingivitis descamativa correspondiente a un LPO a nivel de la encía;

e. Gingivitis descamativa en un LPO situado en la encía;

f. Gingivitis descamativa correspondiente a un LPO a nivel de la encía; g. LPO a nivel del labio inferior. cas y otras regiones como la conjuntiva ocular, nariz, laringe y mucosas genitales (15).

La zona a biopsiar debe ser un área no ulcerada donde se conserve el epitelio y a ser posible que existan estrías (16).

Dentro del cuadro histopatológico del LPO se pueden presentar los siguientes fenómenos:

Se puede observar una hiperqueratosis, que se define como un aumento de espesor de la capa córnea, una acantosis o aumento del número de capas de células granulares donde las papilas se disponen a modo de dientes de sierra (17). La lesión más significativa del LPO es la degeneración hidrópica del estrato basal que es la degeneración de los queratinocitos basales y está relacionada con las patogénesis de la enfermedad. Aparecen los cuerpos de Civatte que son estructuras eosinófílas, redondeadas o lobuladas que a veces contienen núcleos picnóticos o fragmentos nucleares localizados principalmente en la capa basal. Serían los queratinocitos basales que 
Fig. 2. a. LPO erosivo localizado en el dorso de la lengua;

b. LPO erosivo y en placa localizado en el dorso de la c. Liquen erosivo ulcerado y ampolloso en el dorso de la

d. LPO blanco en el suelo de la boca;

e. LPO rojo en la comisura labial y extendido al área retrocomisural;

f. Liquen erosivo ulcerado en el paladar.
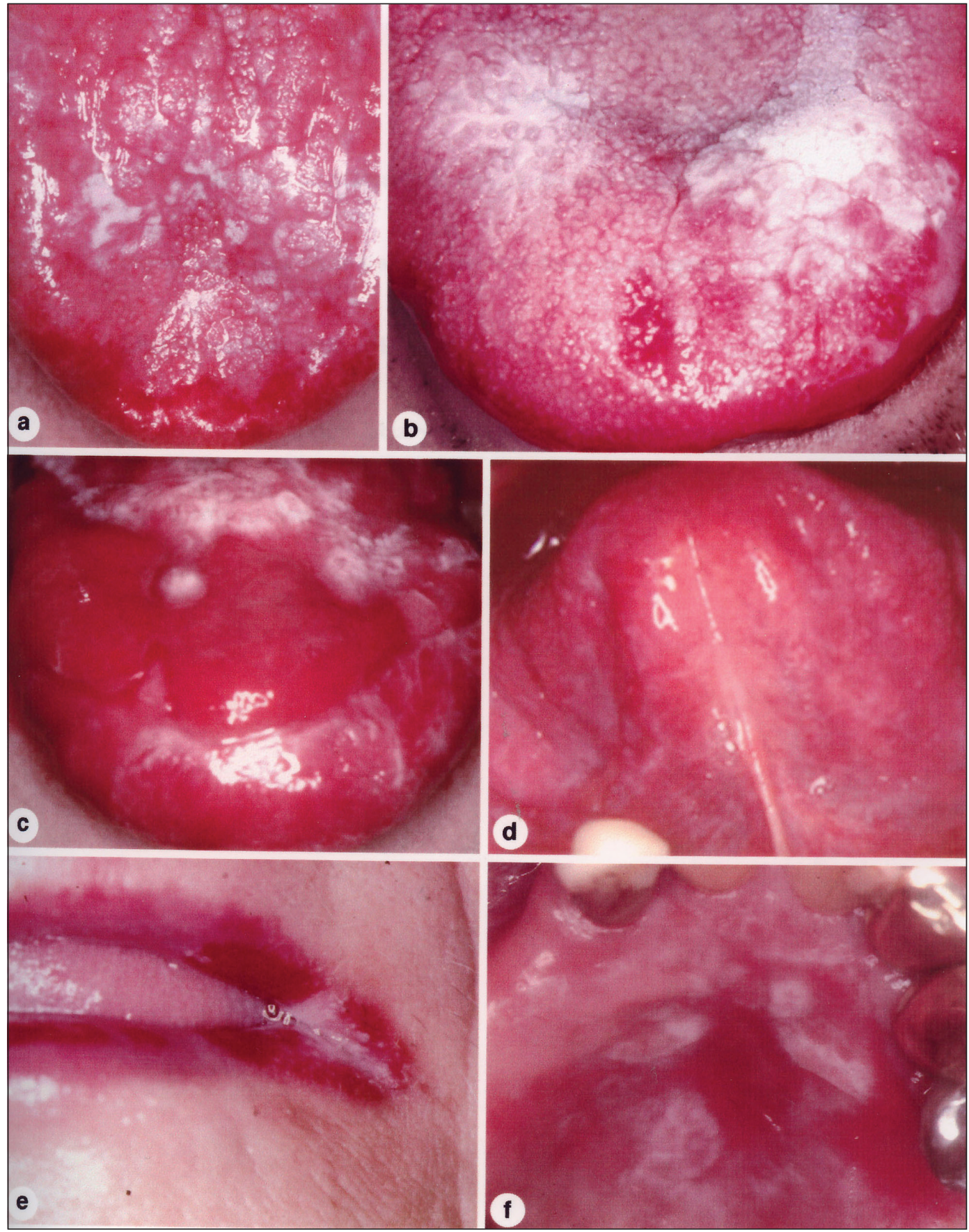

han sufrido necrosis prematura por la degeneración hidrópica de las células basales (18).

En la presencia de un infiltrado inflamatorio subepitelial en banda, principalmente a expensas de linfocitos $\mathrm{T}$, aparecen más los linfocitos colaboradores (CD4) que los supresores (CD8), macrófagos y célu- las de Langerhans (19). Se sugiere que los CD8 migran a través de las roturas de la membrana basal hacia el epitelio (20).

El diagnóstico diferencial se realiza entre el LPO reticular y una serie de lesiones como son las reacciones liquenoides (RL), candidiasis, quemaduras 
químicas, leucoplasia, hiperqueratosis, lupus eritematoso discoide, psoriasis y nevus esponjosos blanco. Se puede hacer inmunofluorescencia directa para demostrar el depósito de fibrinógeno a lo largo de la capa basal en un $85 \%$ y en algunas ocasiones en los cuerpos coloides en un $15 \%$.

Merced a la utilización del microscopio electrónico se puede observar el infiltrado, mitocondrias, aparato de Golgi, degeneración citoplasmática vacuolada, hemidesmososmas etc. (16).

Para algunos autores el LPO es la enfermedad no infecciosa más frecuente de la cavidad oral, y llega a representar el $20 \%$ de los diagnósticos de la práctica de medicina bucal (21).

Su prevalencia en la población general se estima entre $0.2-4 \%$, dependiendo de la población elegida. Algunos autores señalan una prevalencia en España de $0.2-2 \%$. Afecta principalmente a la población adulta entre los 30 y los 70 años, con una edad media de 40-50. Parece tener predilección por el sexo femenino, siendo el $60-70 \%$ mujeres. En éstas suele presentarse entre los 50-59 años y en los hombres entre los 40-50 años de edad (16).

La etiopatogenia es desconocida, aunque hay suficientes datos que permiten suponer que los mecanismos inmunológicos son fundamentales en la iniciación y perpetuación del proceso. Hoy día se acepta que el LPO representa una respuesta inmune mediada por células en la que existe una interacción linfocito-epitelio dirigida contra antígenos de los queratinocitos basales del epitelio que culmina con la degeneración del estrato basal del epitelio.

El liquen cuando se desarrolla atraviesa dos fases: la inductora-proliferativa y la retención-efectora. En la primera se alteran los queratinocitos basales del epitelio por antígenos exógenos o endógenos que reconocen al antígeno a través de su receptor (TCR) lo que sugiere que la reacción autoinmune primaria esta dirigida contra un antígeno desconocido localizado en los gránulos de los queratinocitos del epitelio y no contra los propios queratinocitos orales. Este antígeno es captado y procesado por las células Langerhans y transportados al retículo endoplasmático donde se unen a las proteínas del complejo de histocompatibilidad mayor $(\mathrm{MCH})$ de clase II con los que forman un complejo trimolecular que será transportado a la membrana plasmática (21).

En la fase de retención-efectora se observan los linfocitos unidos a los queratinocitos que ejercen una acción citotóxica.

Las interacciones celulares generadas serán responsables de la cronicidad o extinción de la reacción inflamatoria así como de la agresión. $(22,10)$.

Una mención es obligada a los factores psicológicos en los que la mayoría de los autores están de acuerdo ya que juegan un importante papel en la evolución del LPO. Se ha sugerido que estos pacientes son de un nivel cultural alto sometidos a un estrés elevado, con responsabilidad laboral y familiar importante y en ocasiones muy preocupados por su salud (23). Se ha relacionado el estado de ánimo con la situación inmunológica y los niveles de cortisol especulándose sobre la posibilidad de que una alteración en el estado de ánimo pueda producir un impacto sobre el sistema neuroendocrino, que influiría sobre las subpoblaciones linfocitarias de los pacientes con LPO. Esta relación se encuentra más desarrollada en la variante atrófica-erosiva que en la reticular (24). Los pacientes con LPO tienen un mayor índice de ansiedad, sobre todo en las formas más agresivas de LPO atrófico-erosivo. Por lo tanto además de ser un factor de riesgo la ansiedad también agravaría la lesión (25).

El tabaco es un irritante importante de la mucosa bucal ya que actúa como estímulo nocivo para el epitelio, aunque no se ha podido demostrar que el tabaco actúe como factor etiológico, sí que por sí mismo podría alterar la mucosa ya previamente afectada por el LPO (26).

Actualmente la asociación entre la diabetes mellitus y el LP no se acepta por la mayoría de los autores, aunque se ha especulado que esta asociación podría deberse a la utilización de los antidiabéticos orales u otras drogas, lo que provocaría unas reacciones de tipo liquenoide y no un LP idiomático (27). Más recientemente se han encontrado formas más agresivas (atrófico-erosivas) y localizaciones orales prefe- 
rentes en pacientes con LPO asociado a diabetes mellitus (28).

La posibilidad de asociación entre la hepatitis $C$ y el LP tiene importantes consecuencias clínicas pues si el VHC es más frecuente en pacientes con LP, el diagnóstico de esta entidad nos podía llevar a la comprobación de la existencia de alteraciones hepáticas subyacentes (29). En algunos estudios sí que se observa un aumento de prevalencia de hepatitis $\mathrm{C}$ en pacientes con LP $(30,31)$ aunque todavía no es un tema estrictamente aclarado.

\section{TRATAMIENTO}

El principal problema en el manejo de estos pacientes es su naturaleza crónica con diferentes periodos de actividad y remisión. El tratamiento va enfocado a eliminar las úlceras, aliviar los síntomas y reducir el riesgo de una posible malignización. Lo primero que se debe hacer es eliminar los factores traumáticos próximos a la lesión, tales como restos radiculares, aristas cortantes, ganchos de prótesis etc. Es necesario también eliminar los factores irritantes locales como el tabaco, alcohol y especias. La higiene bucal debe ser exquisita eliminando los depósitos de sarro y tratando de reequilibrar psicológicamente al paciente.

A continuación presentamos diferentes tratamientos utilizados (tabla 1) (32).

Los glucocorticoides siguen siendo los fármacos de elección hoy día en el caso de la variante atróficoerosiva. Son potentes antiinflamatorios inespecíficos e inmunosupresores que van a reestablecer la función de los linfocitos $\mathrm{T}$ supresores. La administración por vía tópica tiene el inconveniente que la absorción es menor que por otras vías utilizándose o como complemento de la vía sistémica o bien en casos de lesiones no muy severas.

Tradicionalmente se presenta en orabase, que es la combinación de gelatina, pectina y carboximetilcelulosa sólida en un gel de polietileno y aceite mineral. Los fármacos más utilizados son el acetónido de triamcinolona al $0,1 \%$ en orabase o en infiltración perilesional a dosis de $30 \mathrm{mg} / \mathrm{ml}$, propionato de
TABLA 1: TRATAMIENTOS DEL LPO (MODIFICADA DE CARROZO Y GANDOLFO 1999)

\section{Corticoesteroides}

- Tópicos:

- Fosfato de betametasona

- Valerato de betametasona

- Propionato de clobetasol

- Acetónido de fluocinolona

- Fluocinonida

- Hemisuccinato de hidrocortisona

- Acetónido de triamcinolona

- Sistémicos:

- Prednisona

- Metilprednisolona

\section{Retinoides}

- Tópicos:

- Fenretinida

- Isotretinoina

- Tretinoina

- Tezarotene

- Sistémicos:

- Acitracina

- Etretinato

- Isotretinoina

- Tretinoina

- Temaroteno

\section{Agentes inmunosupresores}

- Ciclosporina

- Aziatropina

\section{Otros}

- Anfotericina A

- Dietilditiocarbamato

- Dapsona

- Doxiciclina

- Sulfato de hidroxicloroquina

- Levamisol

- Gliciricina

- Griseofulvina

- Eticol

- Enoxaparina 
clobetasol al 0,05\%, acetónido de fluocinolona al $0,025 \%$ en orabase, valerato de betametasona en aerosol y fluocinocida al $0,05 \%$ en orabase. Se recomienda su uso entre 3-5 veces /día después de las comidas durante 4-6 semanas según la evolución de las lesiones. El principal efecto secundario es la sobreinfección por hongos, fundamentalmente por cándidas y por ello algunos autores proponen el uso de antimicóticos paralelamente al uso de corticoides (33).

En casos severos con lesiones de carácter atróficoerosivo intenso se utiliza la vía sistémica con la administración de 40-80 mg/día de prednisona diarios, reduciendo la dosis según se vaya presentando la mejoría del cuadro clínico. En general la respuesta de los pacientes a un ciclo corto de tratamiento es beneficiosa aunque después del mismo suelen recidivar los signos y los síntomas.

Los retinoides actúan sobre el crecimiento y diferenciación celular así como sobre la inflamación reduciendo el infiltrado inflamatorio de linfocitos CD4 y aumentando los macrófagos en lesiones con LPO. Es mejor su uso tópico dado sus efectos secundarios.

La ciclosporina A es un potente inmunosupresor que interfiere con las células $\mathrm{T}$ y las B. Su aplicación es por vía tópica Es necesario una vigilancia estricta del paciente. La aziatropina se usa asociada a corticoides con el fin de aumentar el efecto inmunosupresor.

Otros fármacos como antifúngicos, levamisol, interferón alfa, talidomida etc. no han demostrado efectividad clínica.

\section{BIBLIOGRAFÍA}

1. Dekker Np, Lozada-Nür F, Lagenaur LA, MacPhail LA, Blom CY, Regezi JA. Apoptosis-associated markers in oral lichen planus. J Oral Pathol Med 1997;26:170-5.

2. Neppelberg E, Johannessen Ac, Jonsson R. Apoptosis in oral lichen planus. Eur J Oral 2001; 109:361-4.
3. Walsh LJ, Savage NW, Ishiit T, Symour GJ. Inmunopathogenesis of oral lichen planus. J Oral Pathol Med 1990;19:389-96.

4. Majorana A, Facchetti F, pellegrini W, Sapelli P. Apoptosis-associated markers in oral lichen planus. J Oral Pathol Med 1999;28:47-48.

5. Noda A, Ning Y, Venable SF, Pereira-Smith Om, Smith JR. Cloning of senescent cell-derived inhibitors or DNA synthesis using an expression screen. Exp Cell Res 1994;211:90-8.

6. Bloor BK, Malik FR, Odell EW, Morgan Pr. Quantitative assessment of apoptosis in oral lichen planus. Oral Surg Oral Med Oral Pathol Radiol Endod 1999; 88:187-95.

7. Tobón-Arroyaye SI, Villegas-Acosta FA. Ruiz Restrepo SM, Vieco-Durán M, Restrepo-Misas M, Londoño-López ML. Expresión of caspase-3 and structural changes associated with apoptotic cell death of keratincytes in oral lichen planus. Oral Diseases 2004;10:173-8.

8. Cerero- Lapiedra R, García-Núñez JA, GarcíaPola MJ. Liquen plano oral. RCOE 1997;2:64360.

9. Valente G, Pagano M, Carozo M, Carbone M, Bobba V, Palestra G, Gandolfo S. Sequential immunohstochemical p53 expression in biopses of oral lichen planus undergoing malignant evolution. J Oral Pathol Med 2001; 30:135-40.

10. González Moles MA, Rodriguez-Arcilla A, Ruiz Ávila I, Morales Garcia P. Liquen plano oral. RCOE 2000;5:255-69.

11. Valeron F, Caballero M, Caballero R. Liquen plano oral. Espejo clínico 1999;0:6-11.

12. Carozo M, Broccoletti R, Carbone M, Gandolfo S, Garzino P, Gascio G. Phenotipic análisis of peripheral blood cell immunity in Italian patients with varieties of oral lichen planus Bull Gropu Int Reach Sci Stomatol et Odontol 1996; 39: 33-8 
13. García-Pola MJ, Anitua MJ; Fernandez BE, García JM, López A. Estudio comparativo de la expresión del Ki-67 en el liquen plano oral y leucoplasia oral. Análisis cuantitativo. Medicina oral 2001;6:364-7

14. Van der Meij E, Van der Waal I. Lack of clinicopathologic correlation in the diagnosis of oral lichen planus based on the presently available diagnostic criteria and suggestions for modifications. J Oral Pathol Med 2003;32:50712.

15. Bagan JV, Cerero R. Liquen plano oral. En. Ceballos A. Bermejo A, Aguirre JM, Peñarocha M. Medicina oral. Barcelona: Masson 1995:20219.

16. Blanco A, Gándara JM, Rodríguez A, García A, Rodríguez I. alteraciones bioquímicas y su correlación clínica con el liquen plano oral. Medicina Oral 2000;5:14-25

17. Bascones A, Seoane JM, Aguado A, Quintanilla $\mathrm{JM}^{a}$. Cáncer y precáncer oral. Bases clínico-quirúrgicas y moleculares. Madrid: Ediciones Avances, 2003;157-77.

18. Hirota M, Ito T, Okudela K, Kawabe R, Yazawa T, Hayashi H, Nakatani Y, Fujita K, Kitamura H. Cell proliferation activity and expression of cell cycle regulatory proteins in oral lichen planus. J Oral Pathol Med 2002;31:204-12.

19. White FH, Jin Y, Yang L. Quantitative cellular and nuclear volumetric alterations in epithelium from lichen planus lesions of human buccal mucosa. J Oral Med 1994;23:205-8.

20. Zhou XJ, Sugerman PB, Savage NW, Walsh LJ, Seymour G. Intraepithelial CD8 $+\mathrm{T}$ cells and basement membrane disruption in oral lichen planus. J Oral Pathol Med 2002;31:23-7.

21. Walton LJ, Thornhill Mh, Farthing PM. T cell antigen receptor expression by intraepithelial lymphocytes in oral lichen planus. J Oral Pathol Med 1996;25:534-7
22. Konter U, Kellner I, Hoffmeister B, Sterry W. Induction and upregulationof adhesión receptors in oral and termal lichen planus 1990;19: 25569.

23. García-Pola MJ y Cerero-Lapiedra R. Liquen plano en la mucosa oral. Revista clínica española 1998; vol. 1998,7:448-57.

24. Rödstrom PO, Jontell M, Hakeberg M, Berggren $U$, Lindstedt G. Erosive oral lichen planus and salivary cortisol. J Oral Pathol Med 2001;30: 257-63.

25. García-Pola MJ y Huerta G. Valoración de la ansiedad como factor etiológico del liquen plano oral. Medicina oral 2000; vol. 5,1:7-13.

26. Bagan JV. Liquen plano oral. En: Bascones A, Seoane JM, Aguado A, Suárez JM. Cáncer y precáncer oral.Madrid: Ediciones Avances S.L 2003:155-77.

27. Lozada-Nur F y Miranda C. Oral lichen planus: epidemiology, clinical charactesitics, and associated diseases. Seminars in cutaneous Medicine and Surgery 1997; vol. 16(4):273-7.

28. Romero MA, Seoane J, Varela-Centelles P, DizDios P, García-Pola MJ. Prevalencia de la diabetes mellitus en pacientes con liquen plano oral (LPO). Características clínico-patológicas. Medicina oral 2002;7:121-9.

29. Guijarro B, López A, Hernández G. Aparición de un liquen plano erosivo durante el tratamiento con interferón alfa-2a por una hepatitis C crónica. Medicina oral 2001;6:358-63.

30. Nagao Y, Santa M, Noguchi S, Suzuki H, Mizokami M, Kameyama T, Tanikawa K. GB virus infection in pacients with oral cancer and oral lichen planus. J Oral Pathol Med 1997;26:13841.

31. Bagan JV, Ramón C, González L, Diago M, Milian MA, Cors R, Lloria E, Cardona F, Jiménez Y. Preliminary investigation of the association of 
oral liquen planus and hepatitis C. Oral Surg Oral Med Oral Pathol Oral Radiol Endod 1998;85:532-36.

32. Carrozo M, Gandolfo S. The managment of oral lichen planus 1999;5:196-205.

33. Micó JM, Delgado E, Berini L, Cosme G. Liquen plano bucal: diagnóstico y tratamiento. Quintessence 2001; vol.14,4:247-56.

\section{CORRESPONDENCIA AUTOR}

Prof. Antonio Bascones

Departamento de Medicina y Cirugía Bucofacial Facultad de Odontología.

Universidad Complutense

Plaza Ramón y Cajal, s/n

28040 Madrid, Spain.

E-mail: antbasco@arrakis.es

Teléfono: +34 913942019

Fax: +34 915338064 